\title{
Imaging lipids in living mammalian oocytes and early embryos by coherent Raman scattering microscopy
}

Paola Borri, Josephine Bradley, lestyn Pope, Wolfgang Langbein, Karl Swann

Paola Borri, Josephine Bradley, lestyn Pope, Wolfgang Langbein, Karl Swann, "Imaging lipids in living mammalian oocytes and early embryos by coherent Raman scattering microscopy," Proc. SPIE 10890, Label-free Biomedical Imaging and Sensing (LBIS) 2019, 1089004 (4 March 2019); doi: $10.1117 / 12.2506248$

SPIE. Event: SPIE BiOS, 2019, San Francisco, California, United States 


\title{
Imaging lipids in living mammalian oocytes and early embryos by Coherent Raman Scattering microscopy
}

\author{
Paola Borri ${ }^{\mathrm{a}}$, Josephine Bradley ${ }^{\mathrm{a}}$, Iestyn Pope ${ }^{\mathrm{a}}$, Wolfgang Langbein ${ }^{\mathrm{b}}$, and Karl Swann ${ }^{\mathrm{a}}$ \\ ${ }^{a}$ Cardiff University School of Biosciences, Museum Avenue, Cardiff CF10 3AX, United \\ Kingdom \\ ${ }^{\mathrm{b}}$ Cardiff University School of Physics and Astronomy, The Parade, Cardiff CF24 3AA, United \\ Kingdom
}

\begin{abstract}
Many promising techniques proposed to monitor gamete developmental potential and quality are invasive and not realistically useful in clinical practise. Hence, there is increasing interest in the development of non-invasive imaging methods that can be applied to mammalian eggs and early embryos. Recent studies have shown that mammalian oocyte and embryo viability are closely associated with their metabolic profile, relying entirely on mitochondria as a source of ATP. Fatty acids, stored in intracellular lipid droplets, are an important source of ATP. We have recently demonstrated the use of Coherent Anti-stokes Raman Scattering (CARS) microscopy to allow chemically-specific, label-free imaging of lipid droplets, with high three-dimensional spatial resolution. Here, we summarize our main findings when using CARS to examine the number, size, and 3D spatial distribution of lipid droplets in mouse eggs and early embryos. Quantitative analysis showed statistically significant differences during oocyte maturation and early embryo development. Notably, CARS imaging did not compromise maturation or development. In mouse oocytes that had been subjected to alterations in mitochondrial metabolism we found that the spatial distribution pattern of lipid droplets was also altered. In addition, differences in the chemical composition of lipid droplets in living oocytes matured in media supplemented with different saturated and unsaturated fatty acids were detected using CARS hyperspectral imaging. We also imaged bovine oocytes, and found that lipid droplets appear to be larger and with less spatial aggregation than in mouse oocytes, possibly reflecting the fact that different species metabolise lipids differently. These data suggest that CARS microscopy is a promising non-invasive technique for assessing specific aspects of the metabolic profile of living mammalian eggs and early embryos, which could be potentially linked to their quality and viability.
\end{abstract}

Keywords: Coherent Raman Scattering, nonlinear optical microscopy, mammalian eggs and embryos, lipid metabolism

\section{INTRODUCTION}

Live cell optical microscopy has revolutionized biomedical research over the last 50 years. The use of high resolution fluorescence imaging on cells and embryos in particular has allowed monitoring the localization and function of individual genes, proteins or signalling molecules. In many such cases being label-free is neither essential nor desirable for cell biologists. This is because the use of chemical probes, or fluorescent protein tags, is widespread and provides the required level of contrast and specificity. Albeit photo-bleaching and associated photo-toxicity is generally a limitation, the long term survival of the cells imaged is not always an issue when imaging runs are relatively short, and many of the embryos model systems such as nematode worms, fruit flies and zebrafish are relatively tolerant of long term imaging under visible light excitation.

However, there are other situations in cell biology where fluorescence microscopy presents a significant constraint on the research questions that can be addressed. Studies of mammalian eggs and early (pre-implantation) embryos are a prime example where being non-, or at least minimally -invasive in terms of labeling and imaging, is either of great benefit or else essential.

Further author information: Send correspondence to borrip@cf.ac.uk

Label-free Biomedical Imaging and Sensing (LBIS) 2019, edited by Natan T. Shaked,

Oliver Hayden, Proc. of SPIE Vol. 10890, 1089004 - (c) 2019 SPIE

CCC code: $1605-7422 / 19 / \$ 18 \cdot$ doi: $10.1117 / 12.2506248$

Proc. of SPIE Vol. 10890 1089004-1 
Mammalian eggs and early embryos are widely studied by biologists. Many studies on cow, pig or horse eggs and pre-implantation embryos are designed to improve reproductive technologies. The mouse is the most common model species used because of the many genetic mutant strains available. Mouse eggs and embryos are also one of the most useful models for human eggs and embryos because they have a similar metabolism. For example the same embryo culture medium can be used for culturing mouse and human embryos, and the reagents and plastic-ware used in in vitro fertilization (IVF) clinics is generally quality controlled using mouse embryos. However, one of the problems with mammalian embryos is that they are very sensitive to visible light..$^{1,2}$ Illumination in single photon confocal microscopy can lead to developmental arrest. ${ }^{1}$ The only way to image mammalian embryos with high resolution in a way that is compatible with long term development is to use near-infrared illumination such as with multiphoton fluorescence microscopy. ${ }^{1}$ Another issue with mammalian eggs and embryos is that they show considerable variability in terms of development and this variability is of direct interest. It is often important to be able to analyse and quantify, for example, some metabolic parameters of the embryo and then study how development subsequently proceeds. Hence, methods that can assess the metabolic state of embryos in a way that is quantitative and label free are of great potential value.

Coherent Raman Scattering (CRS) microscope has attracted increasing attention in recent years as a powerful technique offering label-free chemical specificity with high spatial and temporal resolution. CRS microscopy allows for the three dimensional imaging of the chemical composition of living cells with sub-micron spatial resolution, ${ }^{3}$ and it is therefore more specific compared to label free methods based on e.g. phase contrast imaging. It is based on the phenomenon of Raman scattering, namely the interaction of light with vibrating chemical bonds which produces light scattered at a different wavelength (hence color) compared to the incident light. The wavelength shift is a direct signature of the frequency of the vibration, which in turn depends on the type of chemical bond. Although this scattering phenomenon usually produces a very weak signal, it can be enhanced when two short laser pulses are used to coherently drive the vibrations. In this way, the scattered light from all bonds of the same type in the focal volume constructively interferes, generating CRS that can be acquired at high speed for chemically-specific live cell imaging applications. ${ }^{3,4}$ Over the past 10 years, our laboratory has developed and demonstrated a range of label-free CRS microscope set-ups featuring innovative excitation/detection schemes. ${ }^{5-12}$

Specifically to the results shown here, we have developed a multimodal hyperspectral CRS microscope based on a single $5 \mathrm{fs}$ Ti:Sa laser, and associated quantitative chemical imaging algorithms and unsupervised analysis. $^{7,8}$ With this system, we determined the lipid uptake of fixed and living adipose derived human stem cells differentiating into pre-adipocytes, ${ }^{9,13}$ addressed a critical side effect in drug screens, namely, drug-induced lipid storage within hepatic tissue, ${ }^{10}$ and more recently we quantitatively measured masses of lipids, proteins and DNA during cell division. ${ }^{12}$ We also showed that CRS can be used to visualise single non-fluorescing nanodiamonds in cells for the first time. ${ }^{14}$

In this paper, we present some of our main results when applying CRS, and more specifically CARS microscopy, to study the lipid content and spatial distribution in live mammalian oocytes and early embryos.

\section{EXPERIMENTAL SET-UP}

The home-built CARS microscope set-up is based on a single 5 fs laser sources, as described in our previous work, ${ }^{7}$ and schematically shown in Fig. 1. Briefly, pump and Stokes pulses for the CARS excitation are obtained by spectrally separating the broadband laser output with appropriate dichroic beam splitters, resulting in a pump (Stokes) with centre wavelength at $682 \mathrm{~nm}(806 \mathrm{~nm})$ and a bandwidth of $65 \mathrm{~nm}(200 \mathrm{~nm})$. A spectral resolution of $10 \mathrm{~cm}^{-1}$ is achieved through a technique called spectral focusing. This consists in applying an equal linear chirp to pump and Stokes pulses, using glass blocks (SF57 material) of known group velocity dispersion, such that the term driving the vibrational coherence, proportional to their interference, is centered at a well define instantaneous frequency difference (IFD), with an uncertainty (hence spectral resolution) given by the inverse stretched time duration in the ps range. Notably, by tuning the time delay between the pulses, using a motorized delay line, the IFD can be rapidly tuned in the $1200-3800 \mathrm{~cm}^{-1}$ range, which allows for hypespectral imaging without the need to tune the laser source. 
The excitation pulses are coupled via scanning galvo mirrors into a commercial inverted microscope stand (Nikon Ti-U) equipped with differential interference contrast (DIC) optics and a monochrome ORCA-285 Hamamatsu CCD camera. A $1.27 \mathrm{NA}$ x60 water immersion objective (Nikon $\lambda \mathrm{S}$ series) and a 1.35NA oil condenser were used, providing high spatial resolution ( $0.3 \mu \mathrm{m}$ lateral, $0.6 \mu \mathrm{m}$ axial as CARS intensity point-spread function) and forward-CARS collection efficiency. For DIC, a motorized sample stage enabled lateral xy sample movement and a motorized objective focussing enabled axial z movement (Prior ProScan III). CARS at the anti-Stokes frequency is selected by an appropriate band-pass filter (Semrock, $542-585 \mathrm{~nm}$ ) and detected by a photomultiplier tube (Hamamatsu H7422-40). The microscope stand is surrounded by an environmental chamber for mammalian live cell imaging studies at $37^{\circ} \mathrm{C}$ (see Fig. 1B).

SecureSeal imaging spacers (Sigma) of $\sim 120 \mu \mathrm{m}$ thickness and $13 \mathrm{~mm}$ diameter where attached onto standard microscope glass slides. Petroleum jelly was thinly spread around the well of the spacer for an airtight seal. Eggs or embryos were pipetted into a small drop 10-20 $\mu$ l of media, and a coverslip was used to create a sealed chamber. For imaging requiring exchange/addition of media over time, $\mathrm{CO}_{2}$ exposure, or future imaging, living cells/embryos were pipetted into a drop of media covered in oil, on a $25 \mathrm{~mm}$ round glass coverslip placed in an in-house-built imaging dish with a removable glass lid. In this instance a longer working distance $0.72 \mathrm{NA}$ air condenser was used.

\section{OOCYTE MATURATION AND OVULATION}

In most mammals, sexual maturity and sexual cycles are governed by Gonadotrophin-releasing hormone (GnRH) secreted by the hypothalamus. Once puberty is initiated in humans, monthly release of follicle-stimulating hormone (FSH) and luteinising hormone (LH) from the anterior pituitary gland located in the brain stimulates the development of ovarian follicles, resumption of meiosis I and thus the exit of oocytes within the ovary from prophase I (see Fig. 2). Other mammals such as rodents, pigs, cows and sheep enter their oestrous phase several times a year, often affected by season, their cycles differing in length and the number of developing follicles differing with varied litter size. At each cycle, release of FSH from the anterior pituitary induces the growth of a number of follicles. Granulosa cells secrete oestrogen in order to induce proliferation of the endometrial lining of the uterus, and to reduce the amount of mucus surrounding the cervix, in order to allow sperm access. Granulosa cells and the oocyte both secrete glycoproteins to form the Zona Pellucida (ZP), and fluid begins to fill the spaces between granulosa cells, the primary follicle now becoming the secondary follicle. The granulosa cells derive the cumulus cells, and the cumulus oocyte complex (COC) is formed, while stromal cells surrounding the follicle become secretory thecal cells. Of the several developing follicles, only one will reach full maturity, the others rendered useless and degenerating. When the secondary follicle reaches maturity, the release of LH ceases prophase I arrest, and meiosis I is completed. ${ }^{15,16}$ Germinal vesicle breakdown (GVBD) occurs to allow for chromosome segregation, and an asymmetric division is achieved, where the secondary oocyte receives the majority of the cytoplasmic contents, the extruded Polar body (PB) receiving very little. ${ }^{15,17}$ Once meiosis I is completed, the cell enters the second meiotic division. When the developing oocyte reaches metaphase of the second meiotic division (MII), it becomes arrested again, mediated by cell cycle inhibitors.

The timing of oocyte maturation differs greatly between mammalian species, from 6-7 hours in most rodents, to 15-20 hours in human, 16-24 hours in bovine species, 36-40 hours in porcine species, and as long as 40-48 hours in cats. ${ }^{17-21}$ Spontaneous oocyte maturation may also occur in culture if the follicular cells are removed, suggesting a responsibility of granulosa cells to provide or maintain the cell cycle inhibitors in order to maintain prophase I arrest. The timing of spontaneous maturation however is longer than natural hormone-induction. ${ }^{17}$ It is at the MII stage of development that the egg is ovulated from the ovary, a number of hours after undergoing arrest. Only $\sim 400$ eggs are ovulated in one human reproductive lifetime. The surge of LH also causes ovarian contractions which lead to ovulation of the mature egg within its cumulus mass from the ovary and into the fallopian tube. The egg is collected from the ovaries by finger-like projections at the end of the oviducts, called fimbriae, and then is able to move along the tubes, facilitated by cilia lining the epithelium. Once in the fallopian tubes, the egg is wafted down the length of the tubes, where fertilisation by sperm is possible. The MII egg is not released from its arrest until the egg is activated by fertilisation. 

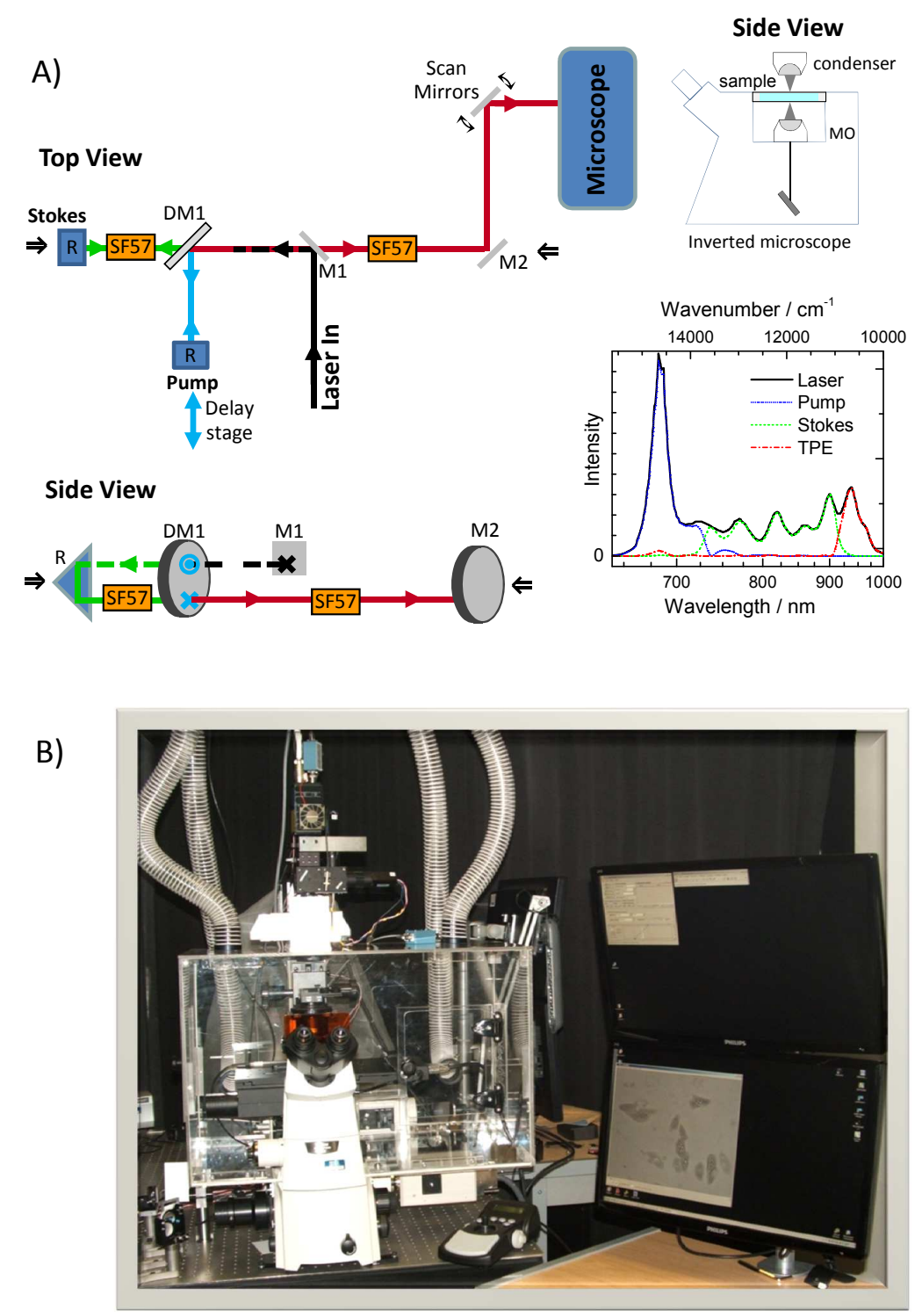

Figure 1. A: Sketch of the microscope set-up. M: mirror; DM: dichroic mirror; SF57: glass blocks; R: reflecting prism. The side view of the optics between by the two indicated arrows shows the beam height difference. The side view of the inverted microscope shows the transmission (forward CARS) geometry via a condenser and a microscope objective (MO) lens. Graph: typical spectra of the laser, pump, and Stokes pulses. The most near infrared part of the laser spectrum can be used for two-photon excitation (TPE) simultaneously with CARS, as described in our previous work . ${ }^{7}$ B: Picture of the commercial microscope stand, equipped with an environmental chamber for mammalian live cell imaging studies.

\subsection{Gamete collection and manipulation}

4-6 week old female MF1 mice were intraperitoneally injected with 5 international units (IU) pregnant mares serum gonadotrophin (PMSG) to mimic the action of FSH and induce ovarian follicle growth. 48hrs later, 10IU human chorionic gonadotrophin (hCG) was injected to mimic LH secretion, to cause superovulation. Oviducts containing mature MII eggs were collected from culled mice 15hrs later, into warmed M2 (embryo-tested, Sigma) medium at $37^{\circ} \mathrm{C}$. Oviducts were punctured with a syringe needle and liberated eggs were collected via mouth pipette. Cumulus cells were removed by brief exposure to $300 \mu \mathrm{g} / \mathrm{ml}$ hyaluronidase. Ovaries containing immature GV oocytes were collected from un-injected culled mice, into warmed M2 medium at $37^{\circ} \mathrm{C}$, containing $100 \mu \mathrm{M}$ isobutyl-1-methylxanthine (IBMX, Sigma) to maintain MI arrest. Ovarian follicles were punctured multiple 


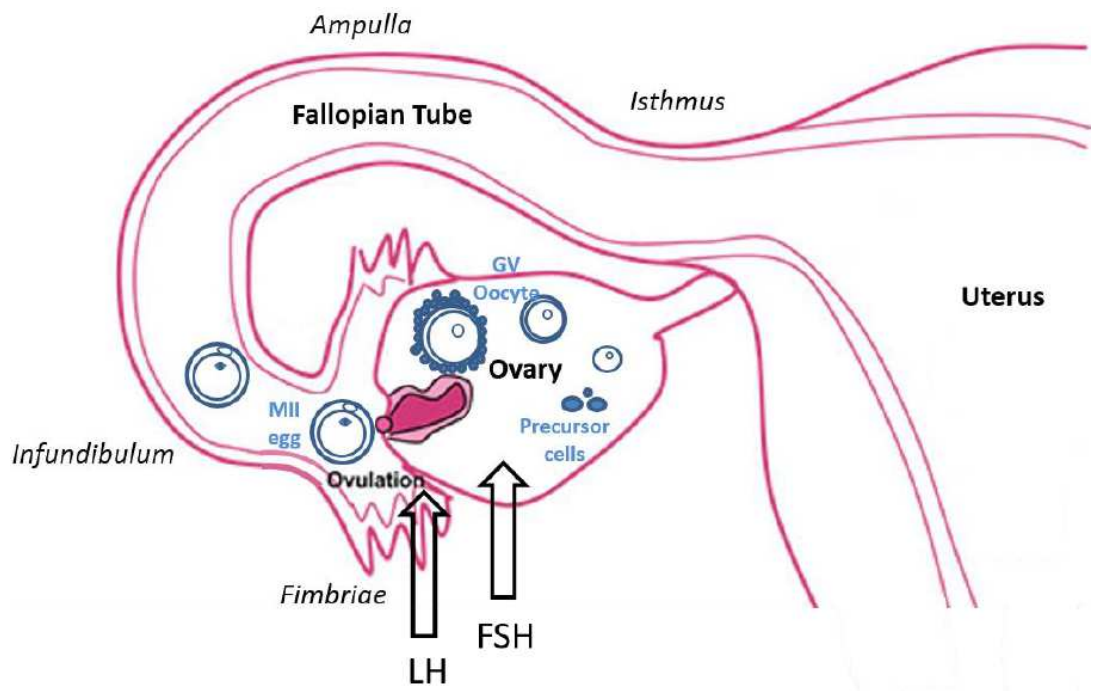

Figure 2. Immature oocytes develop from precursor cells within the ovarian follicles and become arrested at prophase I of the meiotic cell cycle, characterised by a nucleolus contained within a germinal vesicle (GV). At puberty, a surge of luteinising hormone (LH) initiates GV oocyte maturation to metaphase II (MII) of meiosis. Follicle rupture in response to LH causes ovulation of the mature MII egg into the fallopian tube.

times and liberated oocytes were collected via mouth pipette. Cumulus cells were removed by gentle, repeated pipetting, and release from GV arrest was obtained by washing oocytes in multiple drops of M2 medium. During experiments and incubation, oocytes/eggs were kept at $37^{\circ} \mathrm{C}$ in drops of M2 medium (with or without IBMX) covered in mineral oil (embryo-tested, Sigma) to prevent evaporation.

In vivo fertilisation was carried out by mating female MF1 mice with male F1-hybrid mice overnight after hCG injection (48hrs after PMSG), and oviductal zygotes were collected as above 15hrs later. Eggs/embryos were manipulated in all conditions by mouth pipetting, using a fine-bored glass pipette, pulled over a Bunsen flame and connected to plastic tubing with a plastic mouth piece. Pyruvate starvation was carried out by incubating MII eggs in Hepes-buffered KSOM medium (HKSOM) containing 3mg/ml BSA (both Sigma), lacking pyruvate and lactate, for $\sim 6$ hours before imaging.

Bovine ovaries were collected from various culled cattle, and COCs liberated into HEPES-buffered M199 media. Maturation was allowed in supplemented M199 media (10\% serum) at $39^{\circ} \mathrm{C}$, with $5 \% \mathrm{CO} 2$ overnight, before treating with $300 \mu \mathrm{g} / \mathrm{ml}$ hyaluronidase and removing cumulus cells by gentle pipetting with a fine-bore pipette. Bovine eggs were held and imaged in standard M2 medium at $37^{\circ} \mathrm{C}$.

All animals were handled according to UKHome Office regulations, and procedures carried out under a UK Home Office Project Licence.

\section{LIPIDS IN MAMMALIAN EGGS AND EARLY EMBRYOS}

Mammalian eggs and early embryos have particular metabolic features that make them of interest for studying lipids. Like many cell types they generate most of their ATP by oxidative phosphorylation within their mitochondria. However, unlike many cells types they have very low levels of glycolysis and so rely on their mitochondria for essentially all of their ATP generating capacity. ${ }^{22}$ The major substrate for mitochondria in mammalian eggs and early embryos is pyruvate which is present in the oviduct where fertilization and early cell divisions takes place. However, the mitochondria also oxidize fatty acids. Whilst these fatty acids can be found in the medium, their predominant source for oxidation is from intracellular stores in the form of lipid droplets (LDs). ${ }^{23}$ These lipid droplets predominantly consist of triglycerides (three fatty acids esterified to one glycerol molecule). Some mammalian eggs such as those from pigs, cats and dogs contain large numbers of larger sized lipid droplet. These lipid droplets dominate the morphology of the eggs which are so opaque as to appear black 
in a standard transmission light microscope. ${ }^{24}$ Other eggs such as those from mice and humans have much lower lipid content and their cytoplasm appears relatively translucent. Still others, such as those from cows and sheep have an intermediate level of lipid with a cytoplasm that varies in opaqueness from one egg to another.

All mammalian eggs appear to metabolize fatty acids to some extent and the amount of lipid determines how long an early embryo can develop in vitro in the absence of substrates (e.g. pyruvate). There is renewed interest in the lipid content of embryos since it is know that exposure of mouse or cow eggs to free (non-esterified) fatty acids during their maturation phase leads to poor quality embryos that are less likely to develop than control embryos. ${ }^{23,25}$ In addition, in mice there is evidence that there are long term effects on the health of offspring born to obese mothers, and this effect appear to primarily mediated through excess lipid content in the eggs and early embryos. ${ }^{23}$

It is not know why the lipid content of eggs varies so much between species. Nor is it known why a slightly elevated lipid content is detrimental to eggs from mice and humans and yet pig, dog or cat eggs are healthy with a lipid content that is likely to be an order of magnitude greater than that in rodents. The lipid content of eggs can be studied by destructive chemical analysis. ${ }^{24}$ The lipid droplets in eggs and embryos are usually otherwise studied by labelling and imaging them with fluorescence dyes such as Nile red or BODIPY-493/503. ${ }^{23,24}$ However, the specificity of these dyes is unclear and the signals are not necessarily quantitative. More problematic is that these dyes are not compatible with embryo development, i.e. the labeled embryo cannot be followed over time for development. For these reasons we have applied chemically specific CARS microscopy to live mammalian eggs and early embryos.

Fig. 3 shows images of lipid droplets in a representative GV and in vivo matured MII mouse egg. The left panels show DIC images taken as a single z-plane image through the approximately equatorial plane of the oocyte, while the right side shows CARS images at the $\mathrm{CH}_{2}$ symmetric stretch vibrational resonance, as a color coded z-stacks through $50 \mu \mathrm{m}$ depth (see figure caption). The DIC image of the immature oocyte (Fig. 3A) demonstrates its clearly visible GV containing the nucleus, its zona pellucida surrounding the entire cell, and multiple unidentifiable cytoplasmic inclusions. Examination of CARS images throughout the GV oocyte, shows that the lipids within these cells are assembled into roughly uniformly-sized lipid droplets $(\sim 0.5 \mu \mathrm{m}$ diameter) scattered apparently homogenously throughout the cytoplasm, but not within the GV itself (Fig. 3B). In comparison, DIC images of mature MII eggs enables visualisation of common features such as the polar body, and often the metaphase spindle. Again, the cytoplasm is seen to include many unidentifiable vesicles (indicated in the inset, Fig. 3C). CARS imaging of MII eggs reveals $\sim 0.5 \mu \mathrm{m}$ diameter LDs assembled into clusters, with a spatial distribution appearing very different to the distribution seen in GV oocytes (Fig. 3D). These aggregations are formed by several resolvable individual droplets (indicated in the inset, Fig. 3D). Single LDs and LD clusters are distributed throughout the entire volume of the egg, with seemingly no bias towards any side, the centre or the periphery.

In our recent work, these images were analysed quantitatively, in terms of the number, size, and spatial distribution of LDs. ${ }^{26}$ In particular, using in-house developed software, the coordinates of each droplet were determined by fitting the three-dimensional intensity distribution of each droplet within the CARS images. This method enabled us to count the total number of LDs within the measured sample volume, and provided the size of each droplet (width in $x, y$ and $z$ ) as fit parameters. The coordinates were then used to determine whether each individual LD was part of an aggregate (two or more LDs), or was an isolated droplet. A LD was considered part of an aggregate if separated from its nearest neighbour by a distance less than 1.5 times the optical resolution. By then calculating the occurrence of each aggregate size (number of LDs per aggregate), we found that out of all mouse eggs imaged, MII eggs have the most clusters made up of 10 or more droplets. It is also noteworthy that we found that the lipid droplets imaged by CARS in any one plane of focus correlated with small bright dots in the DIC image.

We also examined living mouse embryos by DIC and CARS microscopy, ${ }^{26}$ from the two-cell stage through to blastocyst. By the eight-cell stage, larger droplets are seen, and by the later early-embryo stages (morula and blastocyst) fewer, and much larger LDs are present, varying in size, suggesting fusion of existing droplets as development continues through these stages. Notably, we demonstrated that CARS imaging (under the appropriate conditions) did not impair egg and pre-implantation embryo development. 

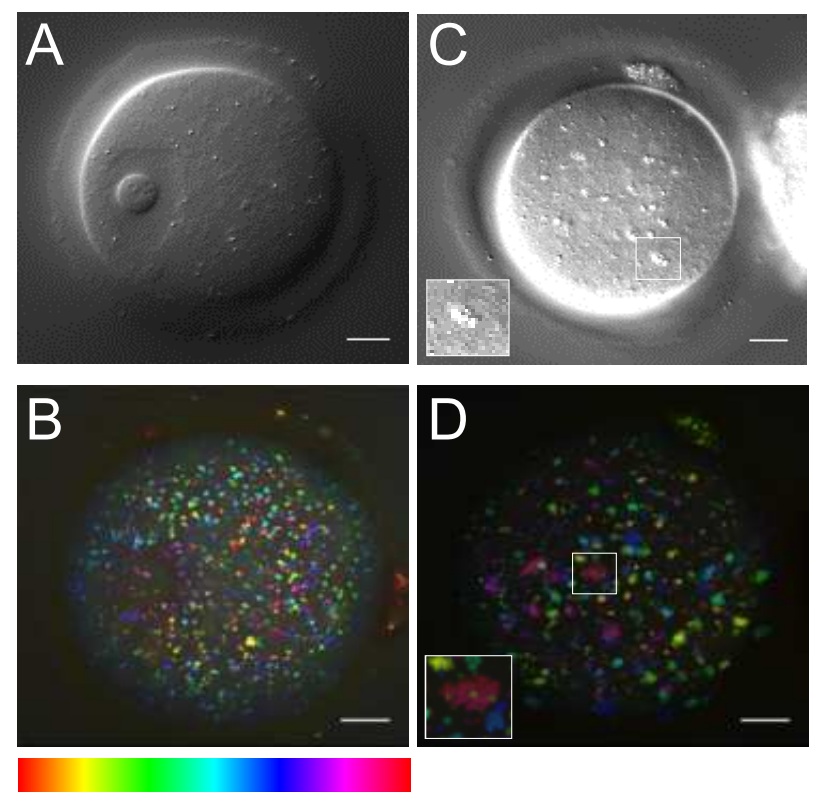

Figure 3. (A) and (C) Single-plane (approximately equatorial) DIC images of representative of (A) immature GV stage oocytes $(\mathrm{n}=\sim 90)$ and $(\mathrm{C})$ mature MII eggs $(\mathrm{n}=\sim 70)$ using a 1.27 NA water objective and a 1.34 NA oil condenser. Inset in (C) shows an unidentified cytoplasmic vesicle cluster. (B) and (D) Depth colour-coded images of CARS z-stacks at wavenumber $2850 \mathrm{~cm}^{-1}$ through the same eggs, showing LDs at these developmental stages. Inset in (D) shows a typical LD cluster seen at this stage. $0.1 \mathrm{x} 0.1 \mu \mathrm{m}$ xy pixel size; $0.5 \mu \mathrm{m}$ z-step; $0.01 \mathrm{~ms}$ pixel dwell time; $\sim 14 \mathrm{~mW}(\sim 9 \mathrm{~mW})$ Pump (Stokes) power at the sample. Scale bars represent $10 \mu \mathrm{m}$; colour bar shows depth colour-coding from $-25 \mu \mathrm{m}$ to $+25 \mu \mathrm{m}$ of $101 \mathrm{z}$-stacks $(0 \mu \mathrm{m}$ being the approximately equatorial plane of the egg), the brightness of each colour is the maximum intensity at each corresponding z-plane. Data from $\geq 2$ trials, using 1-3 mice each.

A clue to the significance of these changes in lipid droplet size and distribution comes from experiments where eggs are placed in a medium devoid of any metabolites - hence they are starved. In such starved eggs the only source of energy for the mitochondria will be fatty acids derived from lipid droplets. With CARS imaging we saw that the aggregates of lipid droplets break up and the droplets are separated and dispersed throughout the cell. ${ }^{26}$ Hence it appears that dispersed lipid droplets represent a state in which fatty acids are being metabolised. It is possible that the spatial separation allows for a larger accessible surface area for fatty acid liberation, and increased contact with mitochondria. Conversely, the aggregates and large droplets could reflect a lack of mitochondrial metabolism of lipids. Further work to demonstrate this hypothesis is in progress and will be published elsewhere.

As well as resolving the number and distribution of lipid droplets we could also gain some insight into the degree of saturation of the fatty acid chains of the triglycerides making up the lipid droplets. Lipids droplets in eggs are known to contain fatty acid chains that show some degree of saturation (i.e. containing some $\mathrm{C}=\mathrm{C}$ bonds). This probably reflects the type of diet eaten by the host. With our hyperspectral CARS microscope we measured CARS spectra of LDs in oocytes and embryos, which were then converted into 'Raman-like' spectra using our data analysis methodology as described in Ref. 8. LDs in the normal mouse oocyte and egg stages, along with the early pre-implantation embryo stages, were observed to be composed of a high proportion of poly-unsaturated fatty acids, and are quite homogeneous in composition across different LDs in the same cell. ${ }^{26}$ Conversely, the variably large LDs found in blastocyst stage mouse embryos appear to be more heterogeneous, some displaying more saturated-like fatty acid spectra. We also found that the fatty acid composition of LDs in eggs reflects that of their culture environment, and it is also shown that the lipid content of LDs can be changed if oocytes are exposed to saturated or unsaturated fatty acids. Eggs were matured in differing concentrations of mono unsaturated oleic acid (OA) and saturated palmitic acid (PA) in order to see how the lipid profile of LDs changed in response to changing environmental fatty acid concentrations. The concentration range used 
were reflective of previously demonstrated effects upon embryo viability, ${ }^{27}$ and PA concentrations likely to be present in the follicular fluid of women over moderate bodyweight. ${ }^{29}$ When supplemented with a particular fatty acid, the chemical composition of LDs resembled that of the fatty acid supplements, providing information that promises to improve our understanding of the effects of OA and PA. Indeed, palmitic acid has been shown to have detrimental effects to the developing egg or embryo, leading to birth defects or embryonic arrest. ${ }^{28,30}$ This embryonic arrest cannot be rescued by the addition of antioxidants, suggesting lipid peroxidation is not the cause of PA-induced damage. ${ }^{30}$ Conversely, oleic acid has been found to have positive effects on eggs and embryos, promoting the formation of triglycerides and their storage in LDs. ${ }^{27,31,32} \mathrm{OA}$ has even been found to reverse the negative effects of palmitic acid and other saturated fatty acids. ${ }^{25,31}$
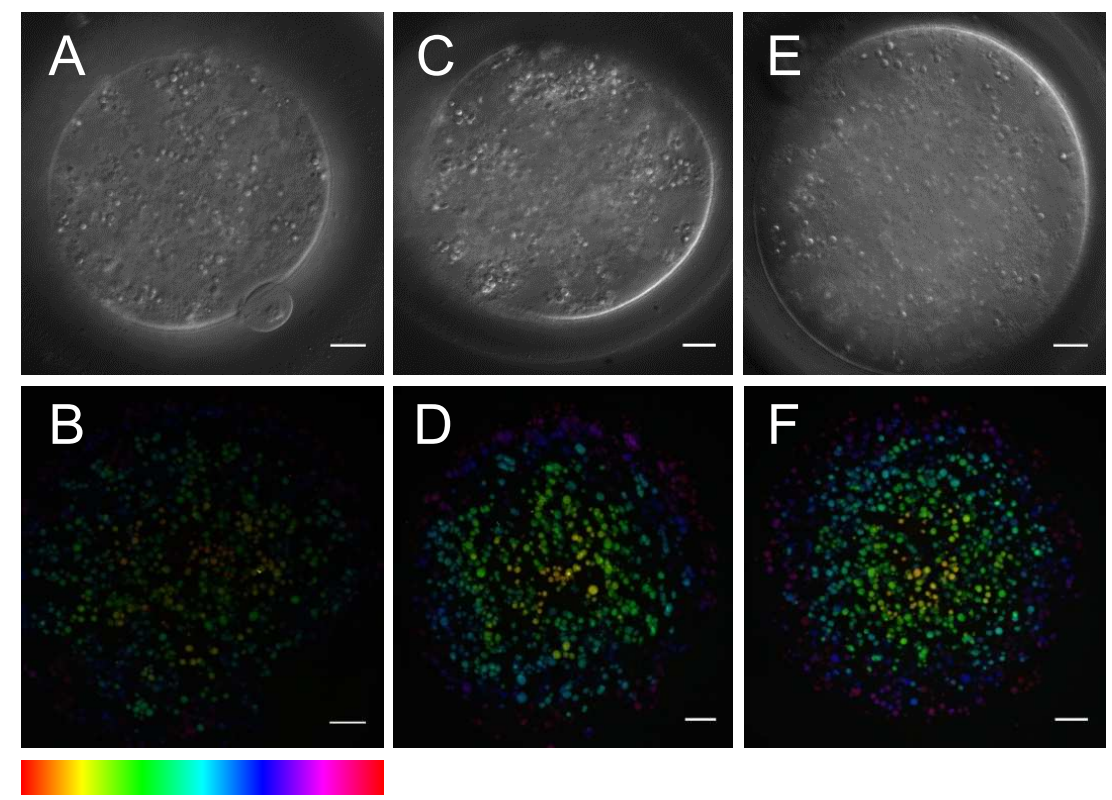

Figure 4. (A), (C) and (E) Single-plane (approximately equatorial) DIC images of representative of MII stage bovine eggs $(\mathrm{n}=13)$ using a $1.27 \mathrm{NA}$ water objective and a $1.34 \mathrm{NA}$ oil condenser. (B), (D) and (F) Depth colour-coded images of CARS z-stacks at wavenumber $2850 \mathrm{~cm}^{-1}$ through the same cells, showing LDs. $0.1 \mathrm{x} 0.1 \mu \mathrm{m}$ xy pixel size; $0.5 \mu \mathrm{m} \mathrm{z}$-step; $0.01 \mathrm{~ms}$ pixel dwell time; $\sim 14 \mathrm{~mW}(\sim 9 \mathrm{~mW})$ Pump (Stokes) power at the sample. Scale bars represent $10 \mu \mathrm{m}$; colour bar shows depth colour-coding from $-25 \mu \mathrm{m}$ to $+25 \mu \mathrm{m}$ of 101 z-stacks $(0 \mu \mathrm{m}$ being the approximately equatorial plane of the egg), the brightness of each colour is the maximum intensity at each corresponding z-plane. Data from $\geq 2$ trials, using 1-3 cows each.

As a comparison to mouse egg LD distribution and content, DIC and CARS imaging were performed on in vitro matured bovine eggs. As seen in DIC images, bovine eggs look similar to those of mice, but are slightly larger (> $100 \mu \mathrm{m}$ diameter as opposed to the $70 \mu \mathrm{m}$ mouse oocyte) and have a more granular-looking cytoplasm (Fig.4 (A), (C), and (E)). CARS images reveal that LDs in mature bovine eggs prove to be larger $(>1 \mu \mathrm{m})$ than those in mouse (Fig.4 (B), (D), and (F)), and non-uniform in size, although not as those seen in murine morula and blastocyst stages. Bovine eggs were not always collected from the same species of cow, and differences were seen between experiments. For example, some eggs were larger than others ( 120-150 $\mu \mathrm{m}$ diameter), some contained larger LDs $(\sim 5 \mu \mathrm{m})$, and some LDs gave a CARS intensity orders of magnitude larger than others.

It is well established that cells of different species metabolise lipids differently, and eggs of species such as dog, pig, cow and sheep contain a larger proportion of LDs and metabolise more lipid. ${ }^{33}$ It appears that the amount of lipid in an egg accurately reflects the amount of lipid metabolism occurring in cells of that species, and that a higher amount of lipid in species which metabolise a large amount of lipid is beneficial to development. Comparison of the LD distribution of mouse eggs with those of alternative species such as bovine thus gives an interesting insight into the different processes occurring in these cells. It is known that there are differing levels of lipid metabolism present in egg cells of different species, so a comparison of LD distributions may give further 
information as to the link between LDs and lipid metabolism. For example, LDs in bovine eggs which appear to be larger with less aggregation than in mice, possibly indicate that lipid metabolism is active. It would be useful and informative to continue this investigation, comparing images seen in this study with further species, such as pigs and humans.

\section{CONCLUSIONS AND OUTLOOK}

In conclusion, we have shown that CARS microscopy is a powerful technique able to quantify the number, size and chemical composition of lipid droplets in living mouse oocytes and early embryos, without apparent photo-damage and impairment of their development. We hypothesize that there is a link between the spatial distribution of lipid droplets and their role in mitochondrial metabolism.

As an outlook, one of the benefits of CARS imaging is that it can be carried out on the same embryos while they develop, as a time course study. This might be most valuable in cow embryos where there is already evidence that the darkness of the cytoplasm correlates with the developmental capacity. CARS microscopy also provides a quantitative way of assessing the impact of high fat diets on eggs by measuring the changes in lipid content, and providing precise metrics on the degree of saturated versus unsaturated fatty acids between eggs from different animals. CARS imaging may also prove useful in studies of human eggs and embryos. Human eggs are variable in appearance and considerable effort has been put into classifying the different grades of cytoplasm in fertilized eggs as a way of assessing embryo viability. Selecting the best embryo for transfer into a prospective mother is major preoccupation of IVF clinics. The current method for embryo selection involves time lapse imaging. This requires collecting bright or dark field images every 5 to 10 minutes over the first few days of embryo development. ${ }^{34}$ The timing of cell divisions, and the time to reach a particular stage of development, are then put into various algorithms to predict which embryo is most likely to develop after implantation. It is still unclear how effective these algorithms are. It is possible that a measurement of the lipid content could contribute to this analysis. It may be unreasonable to expect that IVF clinics will invest in a CARS microscope to carry out embryo selection. However, as noted above, we have observed that the lipid droplets in mouse eggs and embryos coincide with distinctive granules in mouse eggs that are visible with DIC microscopy. It is possible that future analysis of high resolution DIC images of human eggs and embryos may offer a way to assess the lipid content in at least a semi-quantitative manner. The lipid droplet content combined with other aspects of oocyte or embryo morphometrics could make a contribution towards predicting embryo viability.

\section{ACKNOWLEDGMENTS}

This work was funded by the UK BBSRC Research Council (research grant n. BB/P007511/1). PB acknowledges the Royal Society for her Wolfson Research Merit Award (grant n. WM140077). The CARS microscope set-up was funded by the UK BBSRC [grant BB/H006575/1).

\section{REFERENCES}

[1] Squirrell, J. M., Wokosin, D. L., White, J. G., and Bavister, B. D., "Long-term two-photon fluorescence imaging of mammalian embryos without compromising viability," Nat. Biotech. 17, 763-767 (1999).

[2] Takenaka, M., Horiuchi, T., and Yanagimachi, R., "Effects of light on development of mammalian zygotes," Proceedings of the National Academy of Sciences 104(36), 14289-14293 (2007).

[3] Zumbusch, A., Langbein, W., and Borri, P., "Nonlinear vibrational microscopy applied to lipid biology," Progress in Lipid Research 52, 615-632 (2013).

[4] Zhang, C. and Cheng, J.-X., "Perspective: Coherent Raman scattering microscopy, the future is bright," APL Photonics 3(9), 090901 (2018).

[5] Rocha-Mendoza, I., Langbein, W., and Borri, P., "Coherent anti-Stokes Raman microspectroscopy using spectral focusing with glass dispersion," Appl. Phys. Lett. 93, 201103 (2008).

[6] Rocha-Mendoza, I., Langbein, W., Watson, P., and Borri, P., "Differential coherent anti-Stokes Raman scattering microscopy with linearly-chirped femtosecond laser pulses," Opt. Lett. 34, 2258-2260 (2009).

[7] Pope, I., Langbein, W., Watson, P., and Borri, P., "Simultaneous hyperspectral differential-CARS, TPF and SHG microscopy with a single 5 fs Ti:Sa laser," Opt. Express 21, 7096-7106 (2013). 
[8] Masia, F., Glen, A., Stephens, P., Borri, P., and Langbein, W., "Quantitative chemical imaging and unsupervised analysis using hyperspectral coherent anti-Stokes Raman scattering microscopy," Anal. Chem. 85(22), 10820-10828 (2013).

[9] Di Napoli, C., Pope, I., Masia, F., Langbein, W., Watson, P., and Borri, P., "Quantitative spatiotemporal chemical profiling of individual lipid droplets by hyperspectral CARS microscopy in living human adiposederived stem cells," Anal. Chem. 88(7), 3677-3685 (2016).

[10] Masia, F., Pope, I., Watson, P., Langbein, W., and Borri, P., "Bessel-beam hyperspectral CARS microscopy with sparse sampling: Enabling high-content high-throughput label-free quantitative chemical imaging," Anal. Chem. 90(6), 3775-3785 (2018).

[11] Langbein, W., Regan, D., Pope, I., and Borri, P., "Invited article: Heterodyne dual-polarization epi-detected CARS microscopy for chemical and topographic imaging of interfaces," APL Photonics 3(9), 092402 (2018).

[12] Karuna, A., Masia, F., Wiltshire, M., Errington, R., Borri, P., and Langbein, W., "Label-free volumetric quantitative imaging of the human somatic cell division by hyperspectral coherent anti-Stokes Raman scattering," Anal. Chem. 91, 2813-2821 (2019).

[13] Napoli, C. D., Pope, I., Masia, F., Watson, P., Langbein, W., and Borri, P., "Hyperspectral and differential CARS microscopy for quantitative chemical imaging in human adipocytes," Biomed. Opt. Express 5, 13781390 (2014).

[14] Pope, I., Payne, L., Zoriniants, G., Thomas, E., Williams, O., Watson, P., Langbein, W., and Borri, P., "Coherent anti-Stokes Raman scattering microscopy of single nanodiamonds," Nat. Nanotechnol. 9, 940-946 (2014).

[15] Sadler, T. W., Langman's medical embryology, Philadelphia : Lippincott Williams \& Wilkins, 9 ed. (2004).

[16] Downs, S. M., "Specificity of Epidermal Growth Factor Action on Maturation of the Murine Oocyte and Cumulus Oophorus in Vitro1," Biology of Reproduction 41, 371-379 (1989).

[17] Voronina, E. and Wessel, G. M., "The regulation of oocyte maturation," Current Topics in Developmental Biology 58, 53 - 110, Academic Press (2003).

[18] Dominko, T. and First, N. L., "Timing of meiotic progression in bovine oocytes and its effect on early embryo development," Mol. Reprod. Dev. 47(4), 456-467 (1997).

[19] Sirard, M. A., Florman, H. M., , Leibfried-Rutledge, M. L., Barnes, F. L., Sims, M. L., and First, N. L., "Timing of Nuclear Progression and Protein Synthesis Necessary for Meiotic Maturation of Bovne Oocytes1," Biology of Reproduction 40, 1257-1263 (1989).

[20] Johnston, L. A., O'Brien, S. J., and Wildt, D. E., "In vitro maturation and fertilization of domestic cat follicular oocytes," Gamete Res. 24(3), 343-356 (1989).

[21] Hunter, M. G., "Oocyte maturation and ovum quality in pigs," Reviews of reproduction 52, 122-30 (2000).

[22] Dumollard, R., Carroll, J., Duchen, M., Campbell, K., and Swann, K., "Mitochondrial function and redox state in mammalian embryos," Seminars in Cell 83 Developmental Biology 20(3), 346 - 353 (2009).

[23] Dunning, K. R., Russell, D. L., and Robker, R. L., "Lipids and oocyte developmental competence: the role of fatty acids and $\beta$-oxidation," Reproduction 148, 15-17 (2014).

[24] Sturmey, R. G., Reis, A., Leese, H. J., and McEvoy, T. G., "Role of fatty acids in energy provision during oocyte maturation and early embryo development," Reproduction in Domestic Animals 44(s3), 50-58 (2009).

[25] van Hoeck, V., Sturmey, R. G., Bermejo-Alvarez, P., Rizos, D., Gutierrez-Adan, A., Leese, H. J., Bols, P. E. J., and Leroy, J. L. M. R., "Elevated non-esterified fatty acid concentrations during bovine oocyte maturation compromise early embryo physiology," PLOS ONE 6, 1-8 (2011).

[26] Bradley, J., Pope, I., Masia, F., Sanusi, R., Langbein, W., Swann, K., and Borri, P., "Quantitative imaging of lipids in live mouse oocytes and early embryos using CARS microscopy," Development 143(12), 2238-2247 (2016).

[27] Aardema, H., Vos, P. L., Lolicato, F., Roelen, B. A., Knijn, H. M., Vaandrager, A. B., Helms, J. B., and Gadella, B. M., "Oleic Acid Prevents Detrimental Effects of Saturated Fatty Acids on Bovine Oocyte Developmental Competence1," Biology of Reproduction 85, 62-69 (2011).

[28] Jungheim, E. S., Louden, E. D., Chi, M. M.-Y., Frolova, A. I., Riley, J. K., and Moley, K. H., "Preimplantation Exposure of Mouse Embryos to Palmitic Acid Results in Fetal Growth Restriction Followed by Catch-Up Growth in the Offspring1," Biology of Reproduction 85, 678-683 (2011). 
[29] Wu, L., L Russell, D., Norman, R., and L Robker, R., "Endoplasmic reticulum (ER) stress in cumulusoocyte complexes impairs pentraxin-3 secretion, mitochondrial membrane potential $(\Delta \Psi \mathrm{m})$, and embryo development," Molecular endocrinology (Baltimore, Md.) 26, 562-73 (2012).

[30] Nonogaki, T., Noda, Y., Goto, Y., Kishi, J., and Mori, T., "Developmental blockage of mouse embryos caused by fatty acids," Journal of Assisted Reproduction and Genetics 11, 482-488 (1994).

[31] Shaaker, M., Rahimipour, A. R., Nouri, M., Khanaki, K., Darabi, M., Farzadi, L., Shahnazi, V., and Mehdizadeh, A., "Fatty acid composition of human follicular fluid phospholipids and fertilization rate in assisted reproductive techniques.," Iranian biomedical journal 16(3), 162-8 (2012).

[32] Das, S., Mondal, A., and C Elbein, S., "Distinct gene expression profiles characterize cellular responses to palmitate and oleate," Journal of lipid research 51, 2121-31 (2010).

[33] McEvoy, T., Coull, G., Broadbent, P., S. M. Hutchinson, J., and Speake, B., "Fatty acid composition of lipids in immature cattle, pig and sheep oocytes with intact zona pellucida," Journal of reproduction and fertility 118, 163-70 (2000).

[34] Milewski, R. and Ajduk A, "Time-lapse imaging of cleavage cell divisions in embryo quality assessment," Reprodcution 154, R37-R53 (2017). 